\title{
Measurement of differential cross sections in Higgs boson decays to bosons using the ATLAS detector
}

Saskia Falke, on behalf of the ATLAS Collaboration ${ }^{1}$

LAPP, CNRS

\begin{abstract}
With the large $p p$ collision dataset collected by the ATLAS experiment at the Large Hadron Collider at $13 \mathrm{TeV}$, detailed measurements of Higgs boson production can be performed in decays to bosons. This proceeding presents measurements of differential cross sections in Higgs boson decays to two photons or to four leptons, and a comparison to state-of-the-art theory predictions.
\end{abstract}

Keywords:

Higgs, Differential cross sections, Fiducial phase space, $H \rightarrow \gamma \gamma, H \rightarrow Z Z^{*} \rightarrow 4 \ell$

\section{Introduction}

Fiducial and differential measurements present the most model-independent way to measure particle interactions at the Large Hadron Collider (LHC). In contrary to specific tests of the Standard Model (SM) or beyond the SM (BSM) theories, which are highly optimised in sensitivity, differential and fiducial cross sections are based exclusively on the final state properties. Additionally, they are measured within a fiducial phase space that mimics the experimental selection in order to avoid an extrapolation to the full phase space using an underlying theory assumption. Results are generally unfolded, in order to provide measurements independent from the detector resolution or selection efficiencies. Different unfolding techniques can be used depending on the data statistics and event migrations. The method is chosen in order to minimise the uncertainty on the measurement. In the presented analysis, bin-by-bin unfolding is used, which uses independent correction factors computed from simulation in each measured bin. Systematic uncertainties are propagated to these correction factors.

\footnotetext{
${ }^{1}$ Copyright 2018 CERN for the benefit of the ATLAS Collaboration. CC-BY-4.0 license.
}

In each measured region, the cross section can be calculated from the measured signal yield $N_{i}^{\text {sig }}$, the correction factor $c_{i}$, the bin width $\Delta x_{i}$ and the integrated luminosity $\mathcal{L}_{\text {int }}$ as follows:

$$
\frac{d \sigma_{i}^{\mathrm{fid}}}{d x}=\frac{N_{i}^{\text {sig }}}{c_{i} \Delta x_{i} \mathcal{L}_{\text {int }}} .
$$

In measurements with Higgs boson, the measured kinematic distributions generally target different event properties. Measurements of the Higgs boson kinematics (transverse momentum, rapidity, etc.) give information on the perturbative QCD modelling or the Yukawa couplings to the top-, bottom- and charm-quarks. Moreover, the presence of new heavy resonances can be visible in particular in the high momentum tail of the distributions. The number and kinematics of additional jets in the event, produced in association with the Higgs boson allow, for example, to model the contribution from different Higgs boson production modes and of the parton shower. The spin- and CP-properties of the Higgs boson can be inferred by the measurement of angular variables, as for example $\cos \theta^{*}$ or the azimuthal angle between two jets. Higher level variables using information from different final state particles can be used to target the signature of specific SM production pro- 
cesses as for example VBF, when using information on the Higgs boson and two additional jets. Finally, also multi-dimensional differential distributions are interesting to measure; however, these are currently limited by the available statistics in data.

Two Higgs decay channels are currently exploited for the measurement of differential cross sections: $H \rightarrow \gamma \gamma$ and $H \rightarrow Z Z^{*} \rightarrow 4 \ell$. These channels profit from a good subtraction of the background in the whole measured phase space as well as a good mass resolution in the full reconstruction of the decay. Measurements in these two channels with the ATLAS experiment [1] are presented.

\section{Measurements in the $H \rightarrow \gamma \gamma$ channel}

Differential cross sections in the $H \rightarrow \gamma \gamma$ channels have been measured using $80 \mathrm{fb}^{-1}$ of Run 2 data. Diphoton events are reconstructed from two photons passing tight identification and isolation criteria and requirements of minimal transverse momenta scaled by the diphoton invariant mass [2]. A neural network based algorithm allows an excellent reconstruction of the primary vertex. Additional jets (with $p_{\mathrm{T}}^{j}>30 \mathrm{GeV}$ ) and leptons are reconstructed as well. The fiducial phase space at truth level, shown in Table 1 , is defined similar to the experimental selection, in order to minimise the model uncertainty.

The signal extraction in each kinematic bin is performed using a fit to the diphoton invariant mass spectrum. The signal is parametrised using a double-sided Crystal-Ball function whose shape is determined from simulated signal events, while the background is constrained directly on the data, using a smoothly falling function, which is chosen to minimise the bias on the extracted signal.

The results of example differential cross section measurements in the Higgs boson transverse momentum and the transverse momentum of the leading jet are shown in Figure 1 and 2. These measurements are compared to different SM predictions of the gluon-fusion production mode and the best prediction of the other production modes. All three measurements are in good agreement with the SM predictions, with $p$-values of $31 \%$ and $88 \%$ respectively for the different kinematic distributions compared to the prediction of the ggF production mode (matched to a parton shower) calculated at the next-to-next-to-leading order in the strong coupling constant.

In measurements of the $t t H$ production mode in the $H \rightarrow \gamma \gamma$ channel, one of the dominant systematic uncertainties is due to the poor theoretical modelling of the Higgs production in association with heavy flavour

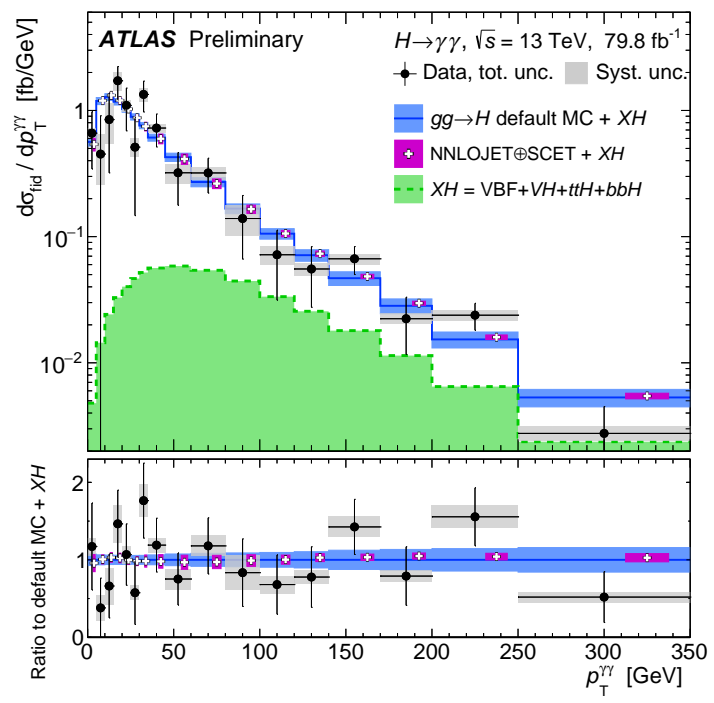

Figure 1: Differential cross sections measured in the $H \rightarrow \gamma \gamma$ channel as a function of the Higgs boson transverse momentum. [2]

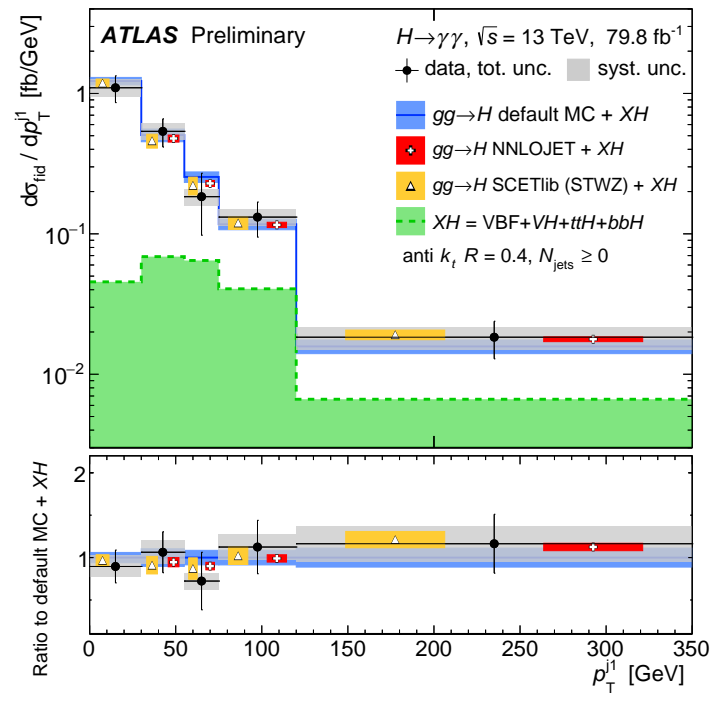

Figure 2: Differential cross sections measured in the $H \rightarrow \gamma \gamma$ channel as a function of the transverse momentum of the leading jet. [2]

jets [3]. In previous measurements of this production mode, a conservative uncertainty of $100 \%$ has been used on this background. Differential measurements of the number of $b$-tagged jets produced in association with the Higgs boson, as shown in Figure 3, can help to constrain the uncertainty on this background in the future.

The main systematic uncertainties on these measurements are the background modelling and the photon energy scale and resolution, impacting the extracted 


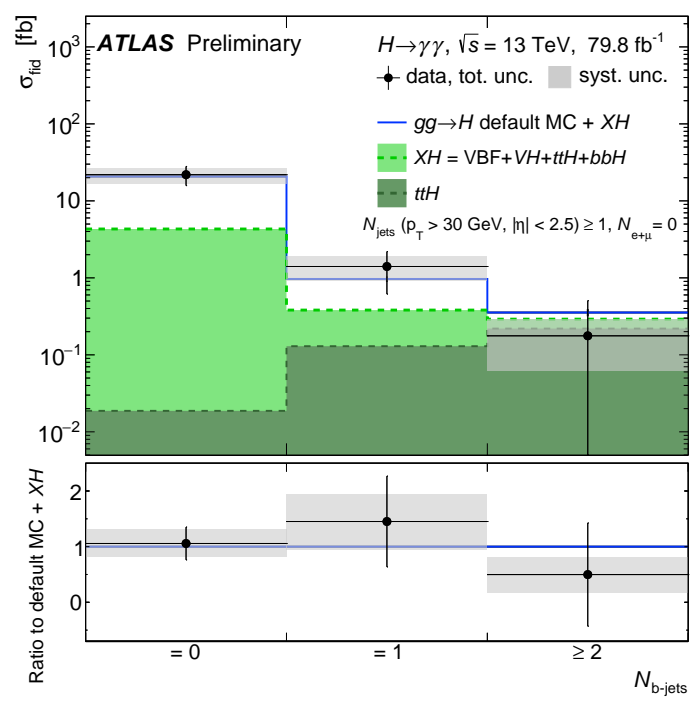

Figure 3: Differential cross sections measured in the $H \rightarrow \gamma \gamma$ channel as a function of the number of $b$-jets produced together with the Higgs boson. [2]

signal yield, as well as the photon identification and isolation efficiencies, which represent uncertainties on the correction factor used for the unfolding process. However, these measurements are currently limited by the limited data statistics and are thus expected to improve by adding more data.

The measured differential cross sections can be interpreted in a model-independent way using an effective field theory (EFT) approach. In particular, the measurement performed in the $H \rightarrow \gamma \gamma$ channel using $36 \mathrm{fb}^{-1}$ of data [4] has been used to constrain three parameters of the SILH basis [5], which modify the couplings of the Higgs boson to gluons and vector bosons. The new physics introduced by these operators is given as follows:

$$
\begin{aligned}
\mathcal{L} & =\bar{c}_{g} O_{g}+\bar{c}_{H W} O_{H W}+\bar{c}_{H B} O_{H B} \\
& +\tilde{c}_{g} \tilde{O}_{g}+\tilde{c}_{H W} \tilde{O}_{H W}+\tilde{c}_{H B} \tilde{O}_{H B}
\end{aligned}
$$

where $O$ and $\tilde{O}$ are $\mathrm{CP}$-odd and a CP-even operators. $c_{i}$ are the Wilson coefficients, which are constrained on the data and correspond to the strength of these operator's contributions. Figure 4 shows the result from a fit to the $\bar{c}_{H W}$ and $\tilde{c}_{H W}$ Wilson coefficients, assuming the same new physics couplings to $W$ and $Z$ boson. The result is in good agreement with the SM expectation.

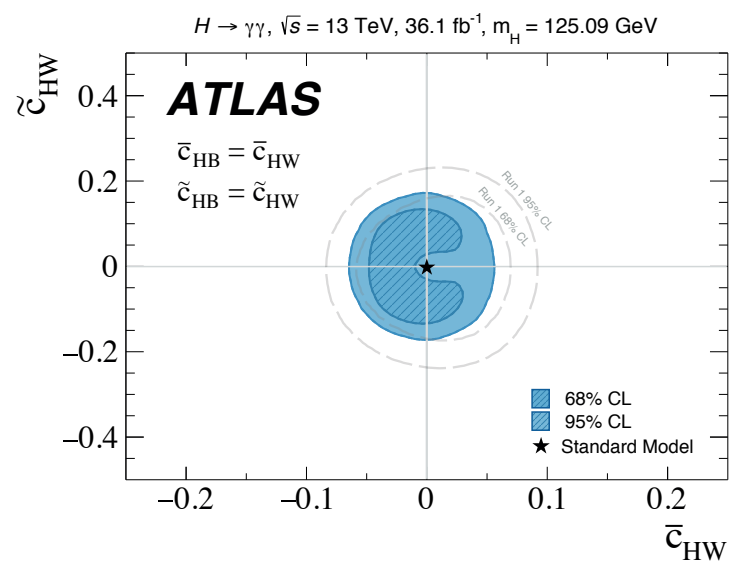

Figure 4: Simultaneous fit to the CP-even and CP-odd $\bar{c}_{H W}$ Wilson coefficients, which modify the couplings of the Higgs to vector bosons. The couplings to $W$ and $Z$ bosons are assumed to be identically modified and the coupling to gluons is set to the SM. [4]

\section{Measurements in the $H \rightarrow Z Z^{*} \rightarrow 4 \ell$ channel}

As in the $H \rightarrow \gamma \gamma$ channel, differential cross sections in the $H \rightarrow 4 \ell$ channels have been measured using $80 \mathrm{fb}^{-1}$ of Run 2 data. The event selection targets the SM $H \rightarrow Z Z^{*}$ decay, where both $Z$ bosons decay into two same flavour, opposite charge light leptons $(\ell=e, \mu)$ with loose identification criteria [6]. Cuts on the dilepton invariant masses assure the compatibility with one on-shell $Z$ boson and one off-shell $Z$ boson. Again, all additional jets with a transverse momentum greater than $30 \mathrm{GeV}$ are reconstructed in addition. The measurement is performed in a fiducial phase space following the experimental selection as defined in Table 2.

The signal is extracted using a template fit to the fourlepton invariant mass distribution, simultaneously in all kinematic bins.

The results of example differential cross section measurements in the Higgs boson transverse momentum and the number of jets are shown in Figures 5 and 6. These measurements are compared to different SM predictions of the gluon-fusion production mode and the best prediction of the other production modes. Again, no significant deviations from the SM predictions can be observed, with $p$-values of $7.8 \%$ and $18 \%$ respectively for the different kinematic distributions compared to the NNLOPS prediction of the $g g F$ production mode.

The main systematic uncertainties on these measurements are related to pileup effects in the electron and muon reconstruction, the luminosity uncertainty, as well as the theoretical modelling of the $Z Z^{*}$ background used in the signal subtraction process. Similar to the 


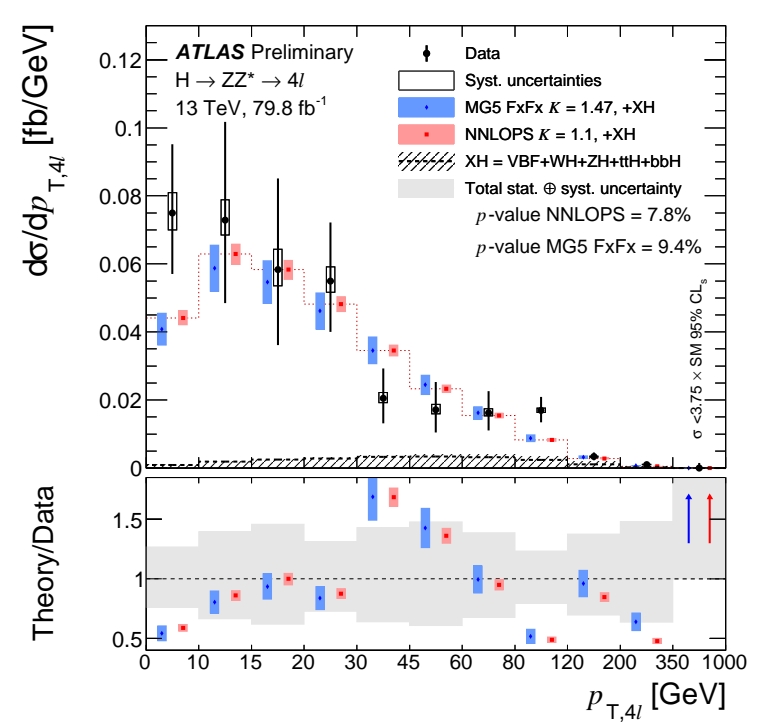

Figure 5: Differential cross sections measured in the $H \rightarrow Z Z^{*} \rightarrow 4 \ell$ channel as a function of the Higgs boson transverse momentum. [6]

$H \rightarrow \gamma \gamma$ channel, this measurement is dominated by statistical uncertainties and will profit from the addition of more data in the future.

The measured differential cross sections can be interpreted using so-called pseudo-observables [7]. The measurement performed in the $H \rightarrow 4 \ell$ channel using $36 \mathrm{fb}^{-1}$ of data [8] has been used to constrain effective coupling modifiers to contact interaction strengths between the Higgs boson and left- or right-handed leptons, as well as a modifier to the coupling of the Higgs boson to the $Z$ boson. An example of such a fit is shown in Figure 7. Again, the results show a good agreement to the SM predictions.

\section{Prospects for future measurements at the HL- LHC}

The High-Luminosity LHC (HL-LHC) will provide around $3000 \mathrm{fb}^{-1}$ of proton-proton collisions at a centreof-mass energy of $\sqrt{s}=14 \mathrm{TeV}$. This large statistics dataset will improve dramatically the precision of differential cross section measurements, allowing to probe the high momentum regime, and possibly exploiting other decay channels. Prospects of the differential cross section measurements in the $H \rightarrow \gamma \gamma$ and $H \rightarrow Z Z^{*} \rightarrow 4 \ell$ channels have been evaluated in the context of a recent Yellow Report [9] with the aim to provide inputs to the European Strategy forum for decisions on future collider projects.

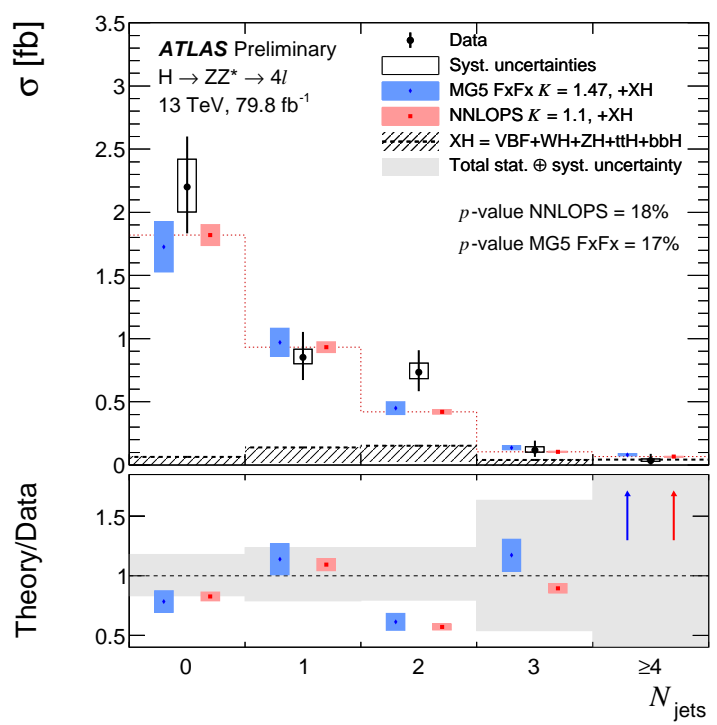

Figure 6: Differential cross sections measured in the $H \rightarrow Z Z^{*} \rightarrow 4 \ell$ channel as a function of the number of jets. [6]

The analyses performed with $80 \mathrm{fb}^{-1}$ of Run 2 data, described in Sections 2 and 3, have been scaled to the integrated luminosity as well as the Higgs boson production cross sections at the HL-LHC. A more realistic projection is obtained in the so-called scenario S2, which additionally introduces a scaling of systematic uncertainties as expected with the HL-LHC conditions. In general, these systematic uncertainties are estimated according to past experience on analysis technique and theory improvements. The upgrade of the ATLAS detectors for the HL-LHC is expected to achieve a similar (or even better) performance as the current detector despite the increased pileup.

Figures 8 and 9 show the projected differential cross section measurements of the Higgs boson transverse momentum and the number of jets, combined in both Higgs decay channels. The projections are presented with the same systematics as in the Run 2 analysis and with more realistic reduced systematic uncertainties. An excellent overall precision with, in most bins, a total uncertainty of below $5 \%$ is obtained. For the first time, a measurement with around $10 \%$ uncertainty is obtained for the high- $p_{\mathrm{T}}^{H}>350 \mathrm{GeV}$ bin, which might provide sensitivity to new heavy resonances. Due to the small model-dependence of the measurements, the results are overall still statistically limited, profiting thus optimally from the large collected dataset. 


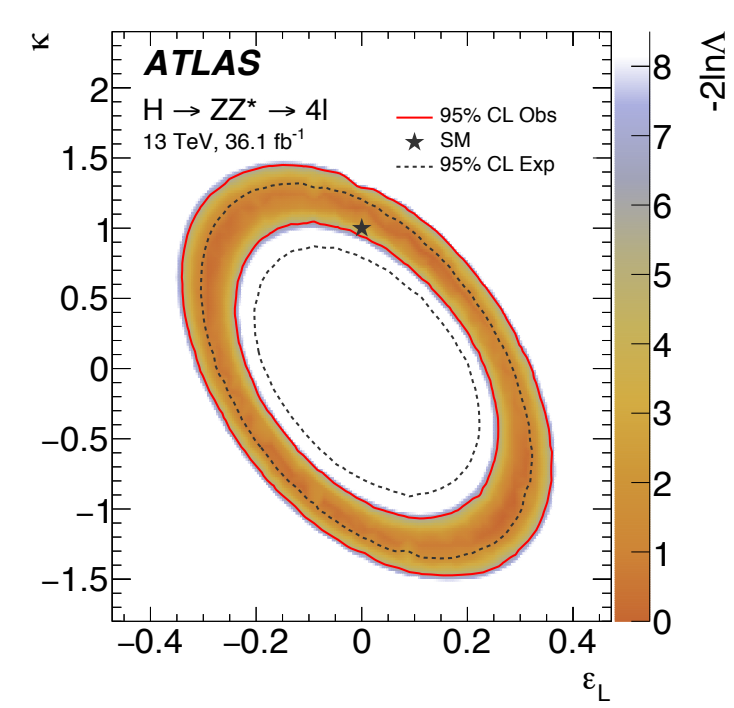

Figure 7: Limits on modified Higgs boson decays within the framework of pseudo-observables. [8]

\section{Conclusion}

Differential measurements of the Higgs boson cross sections represent the most model-independent way to perform measurements of the Higgs properties. The measurements in the $H \rightarrow \gamma \gamma$ and the $H \rightarrow Z Z^{*} \rightarrow 4 \ell$ decay channels using $80 \mathrm{fb}^{-1}$ of ATLAS data were presented. No deviations from the SM predictions were observed, but both measurements are statistically limited and will profit from more data. In the past, measurements in these two channels have been statistically combined [10]. This allows to reduce the statistical limitation of the measurements, but need an extrapolation of the measurements into the full phase space, therefore suffering from larger systematic uncertainties [4, 8]. Measurements with more statistics will allow to constrain a wider range of new physics operators. In particular, measurements at the HL-LHC will allow to constrain the high-transverse momentum regions, which are particularly sensitive to many new physics models.

\section{References}

[1] ATLAS Collaboration, 2008 JINST 3 S08003

[2] ATLAS Collaboration, "Measurements of Higgs boson properties in the diphoton decay channel using $80 \mathrm{fb}^{-1}$ of $p p$ collision data at $\sqrt{s}=13 \mathrm{TeV}$ with the ATLAS detector", July 2018, ATLAS-CONF-2018-028, http://cdsweb.cern.ch/record/2628771

[3] ATLAS Collaboration, "Observation of Higgs boson production in association with a top quark pair at the LHC with the ATLAS detector," Phys. Lett. B 784 (2018) 173, arXiv:1806.00425

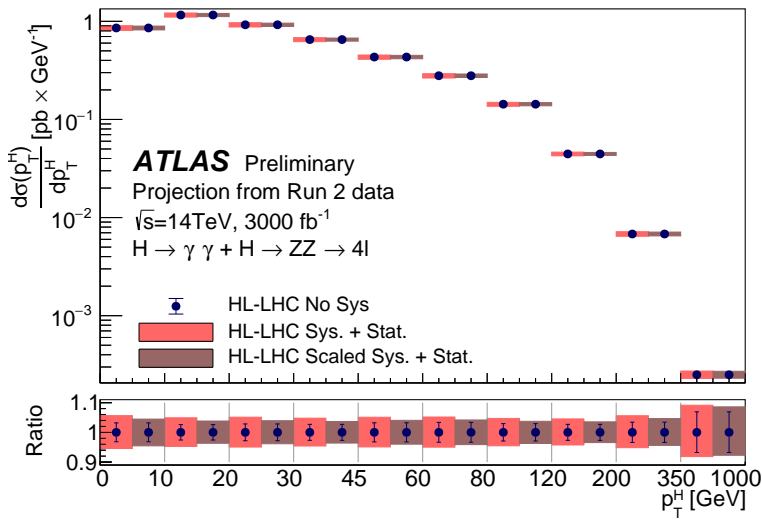

Figure 8: Projection of the combined differential cross section measurement of the Higgs boson transverse momentum in the $H \rightarrow \gamma \gamma$ and $H \rightarrow Z Z^{*} \rightarrow 4 \ell$ channels. [9]

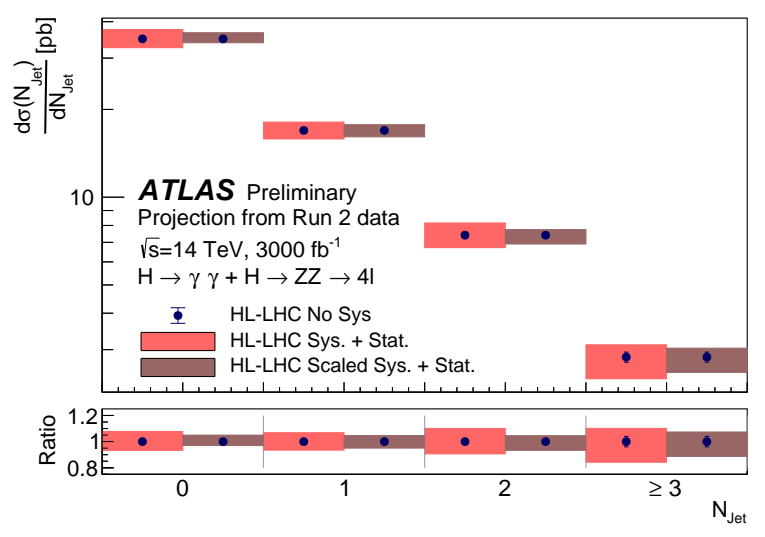

Figure 9: Projection of the combined differential cross section measurement of the number of jets in the $H \rightarrow \gamma \gamma$ and $H \rightarrow Z Z^{*} \rightarrow 4 \ell$ channels. [9]

[4] ATLAS Collaboration, "Measurements of Higgs boson properties in the diphoton decay channel with $36 \mathrm{fb}^{-1}$ of $p p$ collision data at $\sqrt{s}=13 \mathrm{TeV}$ with the ATLAS detector," Phys. Rev. D 98 (2018) 052005, arXiv:1802.04146

[5] G. F. Giudice, C. Grojean, A. Pomarol and R. Rattazzi, "The Strongly-Interacting Light Higgs," JHEP 0706 (2007) 045

[6] ATLAS Collaboration, "Measurements of the Higgs boson production, fiducial and differential cross sections in the $4 \ell$ decay channel at $\sqrt{s}=13 \mathrm{TeV}$ with the ATLAS detector", June 2018, ATLAS-CONF-2018-018, http://cdsweb.cern.ch/record/2621479

[7] M. Gonzalez-Alonso, A. Greljo, G. Isidori and D. Marzocca, "Pseudo-observables in Higgs decays," Eur. Phys. J. C 75 (2015) 128

[8] ATLAS Collaboration, "Measurement of inclusive and differential cross sections in the $H \rightarrow Z Z^{*} \rightarrow 4 \ell$ decay channel in $p p$ collisions at $\sqrt{s}=13 \mathrm{TeV}$ with the ATLAS detector," JHEP 1710 (2017) 132, arXiv:1708.02810

[9] ATLAS Collaboration, "Prospects for differential cross-section measurements of Higgs boson production measured in decays to $Z Z$ and $\gamma \gamma$ with the ATLAS experiment at the High- 
Table 1: Fiducial phase space for measurements in the $H \rightarrow \gamma \gamma$ channel. The definition follows closely the experimental selection of the diphoton events. [2]

\begin{tabular}{ll}
\hline Objects & Definition \\
\hline Photons & $|\eta|<1.37$ or $1.52<|\eta|<2.37, \quad p_{\mathrm{T}}^{\text {iso }, 0.2} / p_{\mathrm{T}}^{\gamma}<0.05$ \\
Jets & anti- $k_{t}, R=0.4, p_{\mathrm{T}}>30 \mathrm{GeV}, \quad|y|<4.4$ \\
$\quad-$ Central jets & $|y|<2.5$ \\
$\quad-b$-jets & $|y|<2.5, \Delta R($ jet, $b$-hadron $)<0.4$ for $b$-hadrons with $p_{\mathrm{T}}>5 \mathrm{GeV}$ \\
Leptons, $\ell=e$ or $\mu$ & electrons: $p_{\mathrm{T}}>10 \mathrm{GeV},|\eta|<2.47($ excluding $1.37<|\eta|<1.52)$ \\
& muons: $p_{\mathrm{T}}>10 \mathrm{GeV},|\eta|<2.7$ \\
\hline Fiducial region & Definition \\
\hline Diphoton fiducial & $N_{\gamma} \geq 2, \quad p_{\mathrm{T}}^{\gamma_{1}}>0.35 \cdot m_{\gamma \gamma}, p_{\mathrm{T}}^{\gamma_{2}}>0.25 \cdot m_{\gamma \gamma}$ \\
$N_{b \text {-jets }}$ measurement & Diphoton fiducial, $N_{\text {jets }}^{\text {Cen }} \geq 1, N_{\text {leptons }}=0$ \\
\hline
\end{tabular}

Table 2: Fiducial phase space for measurements in the $H \rightarrow Z Z^{*} \rightarrow 4 \ell$ channel. The definition follows closely the experimental selection of the diphoton events. [6]

\begin{tabular}{lc}
\hline \hline & Leptons and jets \\
Leptons: & $p_{\mathrm{T}}>5 \mathrm{GeV},|\eta|<2.7$ \\
Jets: & $p_{\mathrm{T}}>30 \mathrm{GeV},|y|<4.4$ \\
remove jets with: & $\Delta R($ jet, $\ell)<0.1$ \\
\hline & Lepton selection and pairing \\
Lepton kinematics: & $p_{\mathrm{T}}>20,15,10 \mathrm{GeV}$ \\
Leading pair $\left(m_{12}\right):$ & SFOS lepton pair with smallest $\left|m_{Z}-m_{\ell \ell}\right|$ \\
Subleading pair $\left(m_{34}\right):$ & remaining SFOS lepton pair with smallest $\left|m_{Z}-m_{\ell \ell}\right|$ \\
\hline \multicolumn{2}{c}{ Event selection $\left(\right.$ at most one quadruplet per event) ${ }_{34}<115 \mathrm{GeV}$} \\
Mass requirements: & $50 \mathrm{GeV}<m_{12}<106 \mathrm{GeV}$ and $12 \mathrm{GeV}<m_{34}$ \\
Lepton separation: & $\Delta R\left(\ell_{i}, \ell_{j}\right)>0.1$ \\
$J / \psi$ veto: & $m\left(\ell_{i}, \ell_{j}\right)>5 \mathrm{GeV}$ for all SFOS lepton pairs \\
Mass window: & $115 \mathrm{GeV}<m_{4 \ell}<130 \mathrm{GeV}$ \\
If extra leptons with $p_{\mathrm{T}}>12 \mathrm{GeV}:$ & Quadruplet with the largest ME \\
\hline \hline
\end{tabular}

Luminosity LHC”, December 2018, ATL-PHYS-PUB-2018040, http://cdsweb.cern.ch/record/2649879

[10] ATLAS Collaboration, "Combined measurement of differential and total cross sections in the $H \rightarrow \gamma \gamma$ and the $H \rightarrow Z Z^{*} \rightarrow$ $4 \ell$ decay channels at $\sqrt{s}=13 \mathrm{TeV}$ with the ATLAS detector,' Phys. Lett. B 786 (2018) 114, arXiv:1805.10197 